\title{
A preliminary study on the efficacy and safety of low level light therapy in the management of cochlear tinnitus: A single blind randomized clinical trial
}

Ji Eun Choi ${ }^{1}$

Min Young Lee ${ }^{1,2}$ Phil-Sang Chung ${ }^{1,2}$ Jae Yun Jung ${ }^{1 *}$

\begin{abstract}
Objectives: To evaluate the efficacy and safety of low-level light therapy (LLLT) using new irradiation parameters for chronic unilateral tinnitus with cochlear dysfunction.

Design: A single-blind, randomized clinical trial

Setting: Tertiary-care hospital center

Participants: Participants who had a history of chronic unilateral tinnitus ( $\geq 3$ months) and pure-tone thresholds greater than $15 \mathrm{~dB}$ (averaged for $3 \mathrm{k}, 4 \mathrm{k}$, and $6 \mathrm{k} \mathrm{Hz}$ ).

Main outcome measures: Numerical rating scales (NRS) measuring loudness, duration, and annoyance, the tinnitus handicap inventory (THI), and psychoacoustical matches of tinnitus loudness and minimum masking levels (MML).

Results: Thirty-eight participants were received either a 100-mW diode laser at 830-nm (TINI group; $\mathrm{n}=19$ ) or placebo (sham group; $\mathrm{n}=19$ ) irradiation through the tympanic membrane. No adverse events were reported during 2 weeks of 10 -interventions (20 minutes/day, five days/week). The NRS measuring duration of tinnitus and psychoacoustical matches of tinnitus loudness significantly decreased over times in the TINI group $(p<0.05)$. However, post-hoc analysis revealed that there was no significant decrease of tinnitus among different time points (baseline, during LLLT, immediately after LLLT, and two weeks after LLLT). There was no placebo effect in the Sham group. Participants who improved the duration by at least one point or improved the loudness matches by more than $5 \mathrm{~dB}$ SL two weeks after LLLT tended to have worse pure-tone thresholds. It may suggest that further study is needed in patients with worse pure-tone thresholds to evaluate the therapeutic efficacy of LLLT.
\end{abstract}

Conclusion: Although this preliminary result is insufficient to support the therapeutic efficacy of new laser device for chronic tinnitus, further study is needed in a large number of selected patients.

Keywords: Tinnitus; low level light therapy; cochlear dysfunction; 830-nm laser.

${ }^{1}$ Department of Otorhinolaryngology, Head and Neck Surgery, Dankook University Hospital, Cheonan, Republic of Korea

'Department of Otorhinolaryngology, Dankook University College of Medicine, Cheonan, Republic of Korea

*Send correspondence to:

Jae Yun Jung MD,PHD.

Department of Otorhinolaryngology,Head and Neck Surgery, Dankook University Hospital, Cheonan, Republic of Korea. E-mail:jikingh2k@gmail.com .Tel:+8241-5563973.

Paper submitted to the ITJ-EM (Editorial Manager System) on June 08, 2019; and Accepted on June 12, 2019 


\section{INTRODUCTION}

Tinnitus is described as the perception of sounds without external or internal auditory stimuli. Tinnitus is one of the most common diseases of the ear and can result in sleep deprivation, loss of concentration, psychological distress, and depression. The prevalence of tinnitus has been reported to range from $19.7 \%$ to $25.3 \%$ in nationwide studies, and some $3 \%$ to $6 \%$ of patients experienced severe discomfort from tinnitus ${ }^{1,2}$. While various therapeutic modalities have been proposed, including medications (antihistamines, sedatives, antiepileptics, antidepressants, antipsychotics, and vasodilators), psychotherapy, and tinnitus retraining therapy, transcranial magnetic stimulation, and transcutaneous electrical stimulation, there is no definitive therapy for tinnitus. Low-level light therapy (LLLT) was proposed as a therapeutic procedure for tinnitus over 20 year's ago $^{3-5}$. Although the exact mechanism of the effect of LLLT on tinnitus is not clearly understood, it has been assumed that low-intensity laser irradiation increases cell proliferation $^{6}$ synthesis of adenosine triphosphate and collagen $^{7,8}$, and the release of various growth factors ${ }^{9}$. It is also assumed to promote local blood flow in the inner ear and activate repair mechanisms through photochemical and photophysical stimulation of the mitochondria in hair cells $\mathbf{s}^{10,11}$. There is still some degree of controversy concerning the effectiveness of LLLT in treating tinnitus. Some studies have shown positive effects ${ }^{10-12}$, but others have found no such effect ${ }^{4,5}$. This discrepancy may be caused by differences in technical parameters (e.g. laser type, wavelength, output power), irradiation targets (e.g., mastoid, external auditory meatus), treatment schedules (e.g., frequency of treatments), and patient selection. Different degrees of laser light transmission to the cochlea could have caused the difference in therapeutic outcomes. Earlier studies evaluating LLLT with a wavelength of 650 $\mathrm{nm}$ reported that there was no significant reduction of symptoms in chronic tinnitus ${ }^{4,5}$. However, recent animal studies have suggested that a diode laser with a wavelength of $810-830 \mathrm{~nm}$ may promote hair cell survival following gentamicin damage in the cochlea and may reduce the salicylate-induced tinnitus ${ }^{13}$. Compared to longer wave length laser (up to 1,000 nm), the infrared laser, especially at wavelengths around $800 \mathrm{~nm}^{14}$, has a lower absorption of water, which enables a greater amount of energy to more deeply penetrate the target tissue. Thus, an $830 \mathrm{~nm}$ laser can transfer energy to the auditory hair cells and auditory nerve without being absorbed by the lymph in the cochlea. Based upon these results, we have developed a trans-tympanic cochlear laser with new irradiation parameters (a wavelength of $830 \mathrm{~nm}$ and output level of $100 \mathrm{mw}$ ) that has more output power than lasers used in earlier studies $\left(40 \mathrm{mw}^{15}\right.$ or $\left.50 \mathrm{mw}^{12}\right)$. This preliminary study evaluated the efficacy and safety of LLLT in comparison with placebo for the improvement of chronic tinnitus with cochlear dysfunction.

\section{MATERIALS AND METHODS}

\section{Ethical considerations}

All participants provided written informed consent before participating in a single-blind, randomized clinical trial. The study protocol was approved by the Dankook University Hospital Institutional Review Board (2016-06-018), and it was performed in accordance with relevant guidelines and regulations. Trial registry can be found at cris.nih. go.kr (Identifier: KCT0002898). The authors confirm that all ongoing and related trials for this intervention are registered.

\section{Subjects and study design}

We conducted a randomized, single-blind, placebocontrolled clinical trial to assess the efficacy of LLLT on participants with cochlear tinnitus. Forty participants with chronic unilateral tinnitus and sensorineural hearing loss were prospectively enrolled. Detailed medical history and examination, pure-tone audiometry, impedance audiometry, and tinnitus scorings were performed for baseline screening. All participants had a history of chronic unilateral tinnitus for more than three months and average pure-tone thresholds greater than $15 \mathrm{~dB}$ for $3 \mathrm{k}, 4 \mathrm{k}$, and $6 \mathrm{k} \mathrm{Hz}$. Exclusion criteria were as follows: the presence of tympanic membrane perforation or ear discharge, conductive hearing loss, any history of exposure to ototoxic drugs or meningitis, any contraindication to laser therapy, any treatment for tinnitus during the previous month. Participants were randomly assigned to either the laser therapy (TINI) or placebo (Sham) group using the permuted block randomization method. SAS Proc Plan was used to generate a random sequence of numbers. The randomization ratio of the two groups was 1:1 with a block size of four, and there were no stratification variables to be considered. The study participants and audiologists who assessed the tinnitus were completely blinded to the groups.

\section{Therapeutic Interventions}

The treatment protocol consisted of 10 interventions for 20 minutes over a period of two weeks (five times a week). Before the intervention, a physician confirmed that the external ear canal was clean. The participants were asked to sit on a chair in a quiet environment and maintain a $30^{\circ}$ head extension and a $60^{\circ}$ head rotation toward the unaffected side. The physician placed a fiberoptic catheter $10-15 \mathrm{~mm}$ in front of the tympanic membrane using an endoscope. During the intervention, all participants and the physician wore laser protective goggles. We used two identically appearing laser devices produced by Won Tech Co.Ltd. (Daejeon, Korea). The TINI device (Won Tech Co. Ltd. Daejeon, Korea) is an indium gallium arsenide (InGaAs) diode laser delivering continuous-wave laser light. This device emits a laser with a wavelength of $830 \mathrm{~nm}$ (infrared radiation) and has a maximum output power of $450 \mathrm{mw}$. This device is certified 
Table 1. Laser treatment parameters.

\begin{tabular}{lc}
\hline Treatment parameters & TINI device \\
\hline Laser type & Diode laser \\
Wavelength $(\mathrm{nm})$ & 830 \\
Pulse rate & Continuous wave \\
Beam diameter $(\mu \mathrm{m})$ & 600 \\
Output power $(\mathrm{mW})$ & 100 \\
Exposure duration sec) & 1,200 \\
Radiant energy $[\mathrm{J}]$ per treatment & 120 \\
Frequency of treatment (times) & 10 \\
Total radiant energy $(\mathrm{J})$ & 1,200 \\
Application technique & $10-15 \mathrm{~mm}$ distance from the \\
\end{tabular}

by the Ministry of Food and Drug Safety (MFDS) (Class III; Approval No. 15-465). The TINI group received irradiation of $100 \mathrm{mw}$ through the tympanic membrane toward the cochlear promontory. The total laser energy applied to the TINI group amounted to 1,200 J. Table 1 shows the laser treatment parameters of the TINI device for tinnitus. The infrared laser was deactivated in the other device. The laser power was measured with a calibrated power meter (S310C, Thorlabs, NJ, USA) in regular intervals (biweekly by the technician) to guarantee consistency. The TINI device was used in accordance with the laser safety regulations of the MFDS.

\section{Efficacy assessments}

We used the following three methods to evaluate tinnitus: the tinnitus Numerical Rating Scale (NRS) for loudness, duration, and annoyance; the Tinnitus Handicap Inventory (THI) questionnaire; and psychoacoustic assessment for pitch, loudness, and Minimum Masking Level (MML). An NRS was compiled for loudness by asking participants, "how loud do you perceive your tinnitus" Their responses were recorded on a scale from $0-10$, with 0 meaning no tinnitus and 10 meaning as loud as imaginable. Duration was assessed by asking, "How long does your tinnitus last," with NRS responses ranging from 0-5 with 0 meaning no tinnitus and 5 meaning always. They were also asked, "How annoying is your tinnitus?" An NRS of 0 meant not annoying, and a response of 10 meant extremely annoying. A Korean validated version of the THI, which consists of a 25-item questionnaire (0$100)^{16}$, was also used to assess the handicap of tinnitus. The NRSs for loudness, duration, and annoyance and the THI questionnaire were assessed in participants before interventions, immediately after 5-interventions, immediately after 10-interventions, and two weeks after 10-interventions. Also, psychoacoustic assessment for pitch, loudness, and MML was performed before the interventions, immediately after 10-interventions, and two weeks after 10 -interventions. Pitch matching was performed by presenting the participants with successive pairs of tones in the unaffected ear, from which they selected the tone that was closest to the tinnitus pitch. A loudness match was determined by balancing the loudness of the tinnitus with the loudness of a tone at pitch matching frequency in the contralateral ear and was expressed in $\mathrm{dB}$ Sensation Level (SL). The MML was established using narrowband noise or white noise. First, the monaural hearing threshold was obtained, and then the wavelength was increased until the participant reported that the tinnitus was inaudible. The loudness was determined using $1-\mathrm{dB}$ steps, and the MML was determined using 5-dB steps.

\section{Statistical analysis}

All data were analysed using SPSS 18.0 (SPSS Inc. Chicago, IL, USA). Statistical comparisons of demographic information and tinnitus characteristics between the TINI and Sham groups were based on independent t-test, Mann-Whitney $U$ test, Pearson's chi-square test, linear-by-linear association test, and Fisher's exact test. To compare tinnitus assessments over time, Friedman test was used. If there was a significant difference over time, post hoc analysis with Wilcoxon signed-rank tests was conducted with a Bonferroni correction applied, resulting in a significance level set at $p<0.0125(0.05 / 4)$ and $p<0.017(0.05 / 3)$ for subjective and objective tinnitus assessments, respectively. To investigate factors associated treatment outcomes of tinnitus, participants was divided into two groups such as respondents and non-respondents. Respondents were defined as participants who improved the tinnitus two weeks after LLLT compared to baseline. Statistical comparisons of age, sex, side, pure-tone thresholds, and baseline pitch matches of tinnitus between respondents and nonrespondents were performed using Mann-Whitney test or Fisher's exact test. A p-value less than or equal to 0.05 was considered statistically significant.

\section{RESULTS}

\section{Study participants}

A total of 38 participants (19 participants in the TINI group and 19 participants in the Sham group) completed this study. One participant in the Sham group was diagnosed with cerebral infarction before intervention and dropped out of the study. Another participant in the TINI group withdrew for personal reasons. Table 2 presents demographic information about the participants and baseline tinnitus characteristics. There were no significant differences in demographic information or baseline tinnitus characteristics between the Sham and TINI groups. Most participants in both the Sham $(n=14)$ and TINI $(n=15)$ groups reported tinnitus pitch matches at $4 \mathrm{khz}$ or above. Participants with high-pitched tinnitus also had similar demographic information and baseline tinnitus characteristics between the two groups.

\section{Assessments of treatment outcomes}

Table 3 shows comparisons of subjective (loudness, duration, annoyance, and THI) and objective (loudness matches and $\mathrm{MML}$ ) tinnitus assessments according to 
Table 2. Demographic information of participants and baseline tinnitus characteristics.

\begin{tabular}{|c|c|c|c|}
\hline Variable & Sham $(n=19)$ & TINI $(n=19)$ & $p$ value \\
\hline \multicolumn{4}{|l|}{ Demographics } \\
\hline Age (years) & $58.4 \pm 11.8$ & $53.3 \pm 12.9$ & $0.211^{\star}$ \\
\hline Sex (male:female) & $11: 08$ & $16: 03$ & $0.074^{\dagger}$ \\
\hline Side (right:left) & 09:10 & $08: 11$ & $0.744^{\dagger}$ \\
\hline PTA $(0.5,1,2$, and $4 \mathrm{kHz}, \mathrm{dB} \mathrm{HL})$ & 48. $0 \pm 21.4$ & $39.1 \pm 24.0$ & $0.467^{\star}$ \\
\hline PTA (4 and 8 kHz, dB HL) & $66.8 \pm 18.4$ & $62.4 \pm 19.1$ & $0.057^{\ddagger}$ \\
\hline \multicolumn{4}{|l|}{ Baseline subjective assessments } \\
\hline Loudness (NRS, 0-10) & $6.6 \pm 1.8$ & $5.6 \pm 1.9$ & $0.172^{\ddagger}$ \\
\hline Duration (NRS, 0-5) & $3.5 \pm 1.0$ & $3.5 \pm 1.0$ & $0.817^{\ddagger}$ \\
\hline Annoyance (NRS, 0-5) & $6.3 \pm 2.1$ & $5.6 \pm 3.0$ & $0.583^{\ddagger}$ \\
\hline THI scores (0-100) & $48.4 \pm 24.4$ & $38.8 \pm 25.4$ & $0.243^{*}$ \\
\hline \multicolumn{4}{|l|}{ Baseline objective assessments } \\
\hline Pitch matches & $5.0 \pm 3.1$ & $5.67 \pm 2.97$ & $0.521^{\ddagger}$ \\
\hline$<4 \mathrm{kHz}$ & 5 & 4 & $0.390^{\pi}$ \\
\hline$\geq 4 \mathrm{kHz}$ & 14 & 15 & \\
\hline Loudness matches (dB SL) & $4.1 \pm 8.2$ & $4.7 \pm 6.9$ & $0.810^{\ddagger}$ \\
\hline Minimum masking level (dB SL) & $-2.7 \pm 11.3$ & $-0.2 \pm 11.4$ & $0.365^{\ddagger}$ \\
\hline
\end{tabular}

NRS: Numerical Rating Scale; THI: Tinnitus Handicap Inventory

Continuous variables were expressed as mean \pm standard deviation; Independent t-test ${ }^{\star}$, Pearson's chi-square test $\dagger$, Mann-Whitney testł, Linearby-Linear Association§, Fisher's exact test $\uparrow$

Table 3. Comparisons of tinnitus assessments over times in Sham and TINI groups.

\begin{tabular}{|c|c|c|c|c|c|c|c|}
\hline Group & Baseline & $\begin{array}{l}\text { Immediately after } \\
\text { 5-interventions }\end{array}$ & $\begin{array}{l}\text { Immediately after } \\
\text { 10-interventions }\end{array}$ & $\begin{array}{l}\text { Two weeks after } \\
\text { 10-interventions }\end{array}$ & $x^{2}$ & $\begin{array}{c}p^{*} \text { for } \\
\text { time }\end{array}$ & $\begin{array}{c}p^{\dagger} \text { for post } \\
\text { hoc analysis }\end{array}$ \\
\hline \multicolumn{8}{|c|}{ Loudness (NRS, 0-10) } \\
\hline Sham & $6.6 \pm 1.8$ & $5.6 \pm 1.8$ & $5.7 \pm 2.0$ & $5.7 \pm 2.0$ & 5.661 & 0.129 & 0.09 \\
\hline TINI & $5.6 \pm 1.9$ & $5.6 \pm 1.9$ & $5.4 \pm 1.8$ & $5.5 \pm 2.0$ & 0.939 & 0.816 & 0.812 \\
\hline \multicolumn{8}{|c|}{ Duration (NRS, 0-5) } \\
\hline Sham & $3.5 \pm 1.0$ & $3.11 \pm 1.2$ & $3.1 \pm 1.2$ & $3.2 \pm 1.0$ & 4.662 & 0.198 & 0.187 \\
\hline TINI & $3.5 \pm 1.0$ & $3.5 \pm 1.0$ & $3.2 \pm 1.2$ & $3.0 \pm 1.3$ & 11.125 & 0.011 & 0.041 \\
\hline \multicolumn{8}{|c|}{$\begin{array}{l}\text { Annoyance (NRS, } \\
0-10)\end{array}$} \\
\hline Sham & $6.3 \pm 2.1$ & $6.0 \pm 2.4$ & $5.3 \pm 2.1$ & $5.3 \pm 2.2$ & 5.216 & 0.157 & 0.127 \\
\hline TINI & $5.6 \pm 3.0$ & $4.8 \pm 2.6$ & $4.8 \pm 3.2$ & $5.4 \pm 2.8$ & 6.467 & 0.091 & 0.621 \\
\hline \multicolumn{8}{|c|}{$\begin{array}{l}\text { THI (Questionnaire, } \\
0-100)\end{array}$} \\
\hline Sham & $\begin{array}{c}48.4 \pm \\
24.4\end{array}$ & $46.7 \pm 22.3$ & $44.2 \pm 22.0$ & $43.4 \pm 20.4$ & 2.798 & 0.424 & 0.678 \\
\hline TINI & $\begin{array}{l}38.8 \pm \\
25.4\end{array}$ & $34.0 \pm 28.3$ & $33.9 \pm 29.1$ & $34.7 \pm 28.2$ & 3.85 & 0.278 & 0.248 \\
\hline \multicolumn{8}{|c|}{$\begin{array}{l}\text { Loudness matches } \\
\text { (dB SL) }\end{array}$} \\
\hline Sham & $4.1 \pm 8.2$ & Not measured & $4.2 \pm 7.2$ & $3.0 \pm 6.2$ & 1.576 & 0.455 & 0.794 \\
\hline TINI & $4.7 \pm 6.9$ & & $3.4 \pm 7.2$ & $1.0 \pm 4.7$ & 6.818 & 0.033 & 0.058 \\
\hline \multicolumn{8}{|c|}{$\begin{array}{l}\text { Minimum masking } \\
\text { level (dB SL) }\end{array}$} \\
\hline Sham & $\begin{array}{c}-2.7 \pm \\
11.3\end{array}$ & Not measured & $-6.4 \pm 6.5$ & $-2.7 \pm 9.6$ & 2.457 & 0.293 & 0.953 \\
\hline TINI & $\begin{array}{l}-0.2 \pm \\
11.4\end{array}$ & & $-2.53 \pm 6.4$ & $-2.2 \pm 7.4$ & 0.391 & 0.822 & 0.875 \\
\hline \multicolumn{8}{|c|}{$\begin{array}{l}\text { NRS: Numerical Rating Scale; THI: Tinnitus Handicap Inventory } \\
\text { *Friedman test } \\
\text { Post hoc analysis with Wilcoxon signed rank test between baseline and two weeks after } 10 \text {-interventions; Data were expressed as mean } \pm \\
\text { standard deviation. Post hoc analysis was conducted with a Bonferroni correction applied, resulting in a significance level set at } p<0.0125(0.05 / 4) \\
\text { and } p<0.017(0.05 / 3) \text { for subjective and objective tinnitus assessments, respectively. Bold indicates a statistically significant difference }\end{array}$} \\
\hline
\end{tabular}

treatment sessions in Sham and TINI groups. There was a statistically significant differences in perceived duration of tinnitus $\left(x^{2}(3)=11.125, p=0.011\right)$ and tinnitus loudness matches $\left(x^{2}(2)=6.818, p=0.033\right)$ depending on treatment sessions in TINI group. Perceived duration of tinnitus in TINI group decreased from $3.5 \pm 1.0$ to $3.0 \pm 1.3$ two weeks after 10-interventions. Tinnitus loudness matches in TINI group also decreased from $4.7 \pm 6.9 \mathrm{~dB}$ SL to 1.0 
Table 4. Factors associated with treatment outcomes of tinnitus two weeks after 10-interventions compared to baseline in TINI group.

\begin{tabular}{|c|c|c|c|c|c|c|}
\hline \multirow[t]{2}{*}{ Variables } & \multicolumn{2}{|c|}{ Duration of tinnitus by NRS } & \multirow[b]{2}{*}{$p$} & \multicolumn{2}{|c|}{ Tinnitus loudness matches } & \multirow{2}{*}{$p$} \\
\hline & Non-respondents & Respondents & & Non-respondents & Respondents & \\
\hline $\mathrm{N}$ & 14 & 5 & & 10 & 8 & \\
\hline Age (years) & $50 \pm 12$ & $63 \pm 11$ & $0.044^{*}$ & $53 \pm 14$ & $56 \pm 14$ & $0.408^{*}$ \\
\hline Sex (male: female) & $12: 02$ & 04:01 & $1.000^{\dagger}$ & 08:02 & 07:01 & $1.000^{\dagger}$ \\
\hline Side (right: left) & 06:08 & $02: 03$ & $1.000^{\dagger}$ & $02: 08$ & 05:08 & $0.145^{\dagger}$ \\
\hline $\begin{array}{l}\text { PTA }(0.5,1,2 \text {, and } 4 \\
\mathrm{kHz}, \mathrm{dB} \mathrm{HL})\end{array}$ & $30 \pm 12$ & $65 \pm 33$ & $0.010^{*}$ & $31 \pm 13$ & $52 \pm 30$ & $0.122^{*}$ \\
\hline $\begin{array}{l}\text { PTA (4 and } 8 \mathrm{kHz}, \mathrm{dB} \\
\mathrm{HL} \text { ) }\end{array}$ & $59 \pm 16$ & $73 \pm 25$ & $0.298^{*}$ & $55 \pm 17$ & $74 \pm 18$ & $0.034^{*}$ \\
\hline $\begin{array}{l}\text { Pitch matches }(<4 \mathrm{kHz} \text { : } \\
\geq 4 \mathrm{kHz})\end{array}$ & 03:10 & 01:04 & $1.000^{\dagger}$ & 03:07 & 01:07 & $0.588^{\dagger}$ \\
\hline
\end{tabular}

NRS: Numerical Rating Scale; Mann-Whitney test*

*Fisher's exact test

Treatment outcomes of tinnitus were compared between baseline and two weeks after 10-interventions. Respondents were defined as participants who improved the duration of tinnitus by at least one point or improved the tinnitus loudness matches by more than 5 dB SL. Continuous variables were expressed as mean \pm standard deviation. Bold indicates a statistically significant difference $(p<0.05)$.

$\pm 4.7 \mathrm{~dB}$ SL two weeks after 10 interventions. Despite on overall decrease in duration of tinnitus and tinnitus loudness matches in TINI group, there were no significant differences between the baseline and two weeks after 10-interventions in post hoc analysis with Wilcoxon signed-rank tests (all $p$ values $>0.05$ ).

\section{Factors associated with treatment outcomes of tinnitus}

Table 4 shows factors associated with treatment outcomes of tinnitus two weeks after 10-interventions compared to baseline. Respondents were defined as participants who improved the duration of tinnitus by at least one point or improved the tinnitus loudness matches by more than $5 \mathrm{~dB}$ SL. In the TINI group, respondents in duration of tinnitus were significantly older $(p=0.044)$ and had significantly worse averaged pure-tone thresholds for $0.5,1,2$, and 4 $\mathrm{kHz}(p=0.010)$ compared to non-respondents. Similarly, respondents in tinnitus loudness matches in TINI group had significantly worse averaged pure-tone threshold for 4 and $8 \mathrm{kHz}(p=0.034)$ compared to non-respondents.

\section{Safety}

All participants tolerated the interventions without serious adverse effects. There was no observable damage to the external ear canal or tympanic membrane during or after LLLT.

\section{DISCUSSION}

This preliminary study evaluated the efficacy and safety of LLLT with new irradiation parameters (diode laser with an $830 \mathrm{~nm}$ wavelength and an output level of $100 \mathrm{mw}$ ) for the treatment of chronic cochlear tinnitus. According to an animal study, only $5-6 \%$ of the output energy can reach the cochlea when an $830 \mathrm{~nm}$ wavelength is used ${ }^{17}$. One way to deliver more laser energy to the cochlea is to increase the output power of the laser. However, the increased irradiated light energy can result in damage to the external auditory canal, tympanic membrane, and inner ear ${ }^{18}$. Previous animal studies using $830 \mathrm{~nm}$ lasers reported a therapeutic effect of tinnitus at an output level of $165 \mathrm{mw}$ (total energy 2,376 J) ${ }^{13}$ and demonstrated safety with an output level of $200 \mathrm{~mW}$ (total energy 5,040 J) ${ }^{18}$. Thus, this study subjected participants to $100 \mathrm{mw}$ of irradiation for 20 minutes with a total of 10 interventions (total energy of 1,200 J). When comparing tinnitus assessments according to treatment sessions in the TINI group, there was a significant overall reduction in perceived duration of tinnitus and tinnitus loudness matches (Table 3). There was no statistically significant improvement of tinnitus in the Sham group. That is, there was no placebo effect to reduce the tinnitus. Although perceived duration of tinnitus in the TINI group significantly decreased from baseline by 0.5 after two weeks for LLLT (from $3.5 \pm 1.0$ to $3.0 \pm 1.3$ ), there were no significant differences among different time points (baseline, during LLLT, immediately after LLLT, and two weeks after LLLT) in post-hoc analysis. Tinnitus loudness matches at pitch matching frequency in the TINI group also significantly decreased from baseline by $3.7 \mathrm{~dB}$ SL after two weeks for LLLT (from $4.7 \pm 6.9 \mathrm{~dB}$ $\mathrm{SL}$ to $1.0 \pm 4.7 \mathrm{~dB} \mathrm{SL}$ ). However, there were no significant differences among different time points in post-hoc analysis. The exact mechanism of tinnitus is still unclear, but hearing damage is thought to be the causal event in most patients ${ }^{19}$. Recent papers have demonstrated a cascade of changes in the central auditory pathway after cochlear damage, including enhanced spontaneous firing rates in various structures of the auditory system, tonotopic reorganization of the auditory cortex, and altered synchronous cortical activity. The logic behind laser-induced photochemical effects for treating tinnitus is its ability to restore the damaged inner ear (hair cells, afferent neuron etc.). In this study, participants who had improvements in perceived duration of tinnitus or tinnitus loudness matches two weeks after LLLT, had significantly worse pure-tone thresholds compared to participants who showed no improvement (Table 4). Previously, Dejakum et al. ${ }^{20}$ applied medium-level $(450 \mathrm{~mW})$ laser therapy with the same wavelength $(830 \mathrm{~nm})$ to our study for chronic tinnitus ${ }^{20}$, but there was no therapeutic effect 
in reducing symptoms of tinnitus. This result may be due to the patient selection. Their study enrolled participants with bilateral or unilateral chronic tinnitus regardless of sensorineural hearing loss. Thus, the LLLT appears to be effective in alleviating chronic tinnitus in patients with worse pure-tone thresholds.

\section{CONCLUSION}

A new laser device with a wavelength of $830 \mathrm{~nm}$ and an output level of $100 \mathrm{mw}$ was safe and effective in reducing the overall duration and loudness matches of tinnitus with cochlear dysfunction. However, there was no significant improvement of tinnitus two weeks after LLLT. This preliminary study provided the possibility of the therapeutic efficacy of the new laser device for chronic tinnitus in patients with worse pure-tone thresholds. Thus, further investigation is still necessary in a larger number of patients with selected criteria.

\section{CONFLICT OF INTEREST}

The Author declares no potential conflict of interest on publishing this paper.

\section{ACKNOWLEDGMENTS}

We thank to So-ol Shin from the Statistical consulting office of Hoseo University in South Korea for helping with statistical analysis

\section{FUNDING}

This study was supported by a grant from the Korea Health Technology R\&D Project, through the Korea Health Industry Development Institute (KHIDI), funded by the Ministry of Health \& Welfare, Republic of Korea (HI15C1524) and the Korea Health Technology R\&D Project, through the Korea Health Industry Development Institute (KHIDI), funded by the Ministry of Health \& Welfare, Republic of Korea (HI15C1524). The programs had no further role in the study design, collection, analysis, or interpretation of data; in the writing of the report; or in the decision to submit the paper for publication.

\section{REFERENCES}

1. Kim HJ, Lee HJ, An SY, Sim S, Park B, Kim SW, et al. Analysis of the prevalence and associated risk factors of tinnitus in adults. PLoS One. 2015; 10:e0127578.

2. Park KH, Lee SH, Koo JW, Park HY, Lee KY, Choi YS, et al. Prevalence and associated factors of tinnitus: data from the Korean National Health and Nutrition Examination Survey 2009-2011. J Epidemiol. 2014;24:417-26.

3. Gungor A, Dogru S, Cincik H, Erkul E, Poyrazoglu E. Effectiveness of transmeatal low power laser irradiation for chronic tinnitus. J Laryngol Otol. 2008;122:447-51.
4. Dehkordi MA, Einolghozati S, Ghasemi SM, Abolbashari S, Meshkat $\mathrm{M}$, Behzad $\mathrm{H}$. Effect of low-level laser therapy in the treatment of cochlear tinnitus: a double-blind, placebo-controlled study. Ear Nose Throat J. 2015;94:32-6.

5. Teggi R, Bellini C, Piccioni LO, Palonta F, Bussi M. Transmeatal lowlevel laser therapy for chronic tinnitus with cochlear dysfunction. Audiol Neurootol. 2009;14:115-20.

6. Van Breugel HH, Bar PR. Power density and exposure time of He$\mathrm{Ne}$ laser irradiation are more important than total energy dose in photo-biomodulation of human fibroblasts in vitro. Lasers Surg Med. 1992;12:528-37.

7. Passarella S, Casamassima E, Molinari S, Pastore D, Quagliariello E, Catalano IM, et al. Increase of proton electrochemical potential and ATP synthesis in rat liver mitochondria irradiated in vitro by helium-neon laser. FEBS Lett. 1984;175:95-9.

8. Redd GK, Stehno BL, Enwemeka CS. Laser photostimulation of collagen production in healing rabbit Achilles tendons. Lasers Surg Med. 1998;22:281-7.

9. Kipshidze N, Nikolaychik V, Keelan MH, Shankar LR, Khanna A, Kornowski R, et al. Low-power helium: neon laser irradiation enhances production of vascular endothelial growth factor and promotes growth of endothelial cells in vitro. Lasers Surg Med. $2001 ; 28: 355-64$.

10. Okhovat A, Berjis $\mathrm{N}$, Okhovat $\mathrm{H}$, Malekpour A, Abtahi H. Low-level laser for treatment of tinnitus: a self-controlled clinical trial. J Res Med Sci. 2011;16:33-8.

11. Huang YY, Chen AC, Carroll JD, Hamblin MR. Biphasic dose response in low level light therapy. Dose Response. 2009;7:358-83.

12. Tauber S, Schorn K, Beyer W, Baumgartner R. Transmeatal Cochlear Laser (TCL) treatment of cochlear dysfunction: a feasibility study for chronic tinnitus. Lasers Med Sci. 2003;18:154-61.

13. Park YM, Na WS, Park IY, Suh MW, Rhee CK, Chung PS, et al. Transcanal laser irradiation reduces tinnitus perception of salicylate treated rat. Neurosci Lett. 2013;544:131-5.

14. Xia N, Wu XY, Wang X, Mou ZX, Wang MQ, Gu X, et al. Pulsed 808$\mathrm{nm}$ infrared laser stimulation of the auditory nerve in guinea pig cochlea. Lasers Med Sci. 2014;29:343-9.

15. Nakashima $T$, Ueda $H$, Misawa $H$, Suzuki T, Tominaga $M$, Ito $A$, et al. Transmeatal low-power laser irradiation for tinnitus. Otol Neurotol. 2002;23:296-300.

16. Newman CW, Jacobson GP, Spitzer JB. Development of the Tinnitus Handicap Inventory. Arch Otolaryngol Head Neck Surg. 1996;122:143-8.

17. Rhee CK, He P, Jung JY, Ahn JC, Chung PS, Lee MY, et al. Effect of low-level laser treatment on cochlea hair-cell recovery after ototoxic hearing loss. J Biomed Opt. 2013;18:128003.

18. Moon TH, Lee MY, Jung JY, Ahn JC, Chang SY, Chung PS, et al. Safety assessment of trans-tympanic photo biomodulation. Lasers Med Sci. 2016;31(2):323-33.

19. Weisz N, Hartmann T, Dohrmann K, Schlee W, Norena A. Highfrequency tinnitus without hearing loss does not mean absence of deafferentation. Hear Res. 2006;222:108-14.

20. Dejakum K, Piegger J, Plewka C, Gunkel A, Thumfart W, Kudaibergenova $\mathrm{S}$, et al. Medium-level laser in chronic tinnitus treatment. Biomed Res Int. 2013;2013:324-34. 